Palavras chave: Semivariograma escalonado Continuidade espacial Inventário florestal Técnicas de amostragem

Histórico: Recebido 06/02/2013 Aceito 14/09/2015

Keywords: Scaled semivariogram Spatial continuity Forest inventory Sampling techniques

Correspondência: isabelcarolinadelima@yahoo.com.br

DOI: 10.1590/01047760201521041824
Isabel Carolina de Lima Guedes', José Márcio de Mello², Eduarda Martiniano de Oliveira Silveira², Carlos Rogério de Mello², Aliny Aparecida dos Reis², Lucas Rezende Gomide ${ }^{2}$

\section{CONTINUIDADE ESPACIAL DE CARACTERÍSTICAS DENDROMÉTRICAS EM POVOAMENTOS CLONAIS DE EUCALYPTUS SP. AVALIADA AO LONGO DO TEMPO}

RESUMO: O objetivo deste estudo foi avaliar a continuidade espacial ao longo do tempo (para 5 idades consecutivas) das características dendrométricas altura dominante média, volume e incremento médio anual em povoamentos clonais de Eucalyptus sp no estado de Minas Geais. A área foi plantada em 2003, perfazendo um total de I.072,6 hectares. Os dados foram oriundos de um conjunto de 116 parcelas permanentes, onde foi realizado inventário florestal sucessivo entre os anos de 2006 e 2010. Aos semivariogramas experimentais, foram ajustados os modelos esférico, exponencial e gaussiano pelo Método dos Mínimos Quadrados Ponderados, para cada idade, selecionando-se o mais adequado com base no erro médio reduzido e desvio padrão do erro médio reduzido da validação cruzada. O comportamento dos semivariogramas foram comparados entre as respectivas medições com base no plotagem dos mesmos de forma escalonada, permitindo avaliar se a estrutura espacial foi alterada com a idade do plantio. $O$ modelo exponencial apresentou-se como $\circ$ de melhor ajuste e todas as características apresentaram-se estruturadas espacialmente, com os modelos espaciais sendo semelhantes entre as idades do povoamento e para as características estudadas. Foi possível verificar que o grau de continuidade espacial se manteve ao longo dos anos para as características avaliadas e que o semivariograma escalonado demonstrou que a estrutura espacial das características é semelhante entre as diferentes idades do povoamento. Os resultados evidenciam que a utilização de métodos geoestatísticos para avaliar o crescimento de povoamentos de eucalipto ao longo do tempo, consiste de uma importante ferramenta de planejamento, permitindo um melhor acompanhamento e uma predição mais precisa do volume de madeira da floresta, levando em consideração a estrutura de dependência espacial.

\section{SPATIAL CONTINUITY OF DENDROMETRIC CHARACTERISTICS IN CLONAL CULTIVATED EUCALYPTUS SP. TRHOUGHOUT THE TIME}

ABSTRACT: The aim of this study was to evaluate the spatial continuity of the dendrometric characteristics average dominant height, volume and mean annual increment, throughout five different continuous ages. The experimental area was planted in 2003, accounting for 1,072.6 hectares. The data sets were derived from 116 permanent plots where successive forest inventory was carried out between 2006 and 2010. Using least squares weighted method, spherical, exponential and gaussian semivariogram models were fitted to the experimental semivariogram. To select the best model, reduced average error and standard deviation of the mean reduced error were analyzed. The spatial continuity assessment at along the time was carried out by comparison of the scaled semivariograms plotted considering the data sets from each age. Exponential semivariogram model has presented the best fitting and all the dendrometric characteristics has presented spatial continuity. The evaluated models were similar for each age and the studied characteristics as well. The results obtained with this study show that the use of geostatistical procedures to evaluate the growth of the trees throughout the time is an important planning tool, allowing a better management and prediction of wood volume in the forest.

' Plantar - Belo Horizonte, Minas Gerais, Brasil

2 Universidade Federal de Lavras - Lavras, Minas Gerais, Brasil 


\section{INTRODUÇÃO}

As informações geradas pelo inventário florestal contínuo alimentam o planejamento estratégico e influenciam diretamente as tomadas de decisão na gestão dos ativos florestais nas empresas do setor florestal brasileiro (GUEDES et al., 20I2). Todo e qualquer ganho, seja na produtividade da floresta, na redução de custo na cadeia produtiva ou, ainda, na melhoria das estimativas de produção da matéria-prima, pode contribuir com o sucesso do empreendimento florestal.

Tradicionalmente nas empresas florestais, o processamento dos inventários é realizado com base nos conceitos da estatística clássica, onde as variações espaciais de uma determinada característica são tidas como independentes, desconsiderando-se as correlações espaciais que possam existir entre as unidades amostrais, mesmo quando estas estão presentes. Contudo, diversos estudos mostraram que as variáveis dendrométricas apresentam-se espacialmente estruturadas, de modo que estas relações não devem ser desconsideradas nas análises estatísticas (ALVARENGA et al., 2012; GUEDES et al., 2012; ASSIS et al., 2009; MELLO et al., 2009; KANEGAE JÚNIOR et al., 2007; KANEGAE JÚNIOR et al., 2006; MELLO et al., 2006; MELLO et al., 2005a; MELLO et al., 2005b).

A presença da correlação espacial entre as unidades amostrais pode ser diagnosticada através do semivariograma experimental ou semivariograma empírico. O semivariograma é um método geoestatístico de caracterização da continuidade espacial de variáveis regionalizadas que exige hipóteses de estacionaridade menos restritivas, como a hipótese intrínseca (RIBEIRO JÚNIOR, 1995).

A componente espacial, quando incorporada nas análises, contribui de forma expressiva para a melhoria da precisão das inferências estatísticas realizadas para a estimativa da característica dendrométrica de interesse (ALVARENGA et al., 2012; GUEDES et al., 2012; ASSIS et al., 2009; MELLO et al., 2009; KANEGAE JÚNIOR et al., 2007; MELLO et al., 2005a).

No entanto, o comportamento da estrutura de dependência espacial pode ser diferenciado para um mesmo plantio ao longo do tempo, significando que à medida que a floresta cresce, a continuidade espacial também pode ser alterada. Este fato evidencia a necessidade de uma análise da continuidade espacial em diferentes ocasiões no tempo, verificando se os semivariogramas teóricos mantêm seus parâmetros estatisticamente parecidos. A continuidade da estrutura de dependência espacial ao longo do tempo permite que análises baseadas em métodos espaciais possam ser utilizados nas diferentes medições, subsidiando o planejamento e melhorando a predição do volume de madeira.

A incorporação da análise da continuidade espacial ao longo do tempo no processo preditivo do crescimento da floresta pode contribuir de forma significativa com as estimativas das variáveis dendrométricas, tornando o inventário mais preciso e passível de previsão do crescimento e da produtividade da floresta em ocasiões futuras, especialmente com redução de custos por meio da estratificação em idades precoce.

Nesse sentido, o objetivo deste estudo foi avaliar a continuidade espacial das características dendrométricas altura dominante média, volume e incremento médio anual (IMA), considerando 5 idades consecutivas de um plantio clonal de Eucalyptus sp. no estado de Minas Gerais.

\section{MATERIAL E MÉTODOS}

\section{Descrição da área de estudos}

A área de estudo corresponde aos povoamentos clonais de Eucalyptus sp., pertencentes à empresa Zanini Florestal, uma das empresas do Grupo Plantar, localizados no município de Morada Nova de Minas, estado de Minas Gerais.

Os solos predominantes são o Latossolo Vermelho distrófico $A$ fraco/moderado, textura argilosa e o Latossolo Vermelho-amarelo distrófico A fraco/ moderado, textura argilosa; ambos fase cerrado, relevo plano e suave ondulado.

O clima da região, segundo a classificação de Köppen, é do tipo Aw e se caracteriza como tropical úmido de savana, tendo no mês mais quente e no mês mais frio, temperaturas médias de $25,1^{\circ} \mathrm{C}$ e $20,9^{\circ} \mathrm{C}$, respectivamente, e precipitação média anual de $1.222 \mathrm{~mm}$ (ANTUNES, 1986).

\section{Descrição da base de dados}

Este estudo foi desenvolvido em um conjunto de 61 talhões plantados entre 02/07/2003 e I5/I2/2003, em espaçamento de $9 \mathrm{~m}^{2}$ por planta, totalizando uma área de 1.072,6 hectares. Os dados foram oriundos de informações obtidas de um conjunto de 116 parcelas permanentes, onde foi 
realizado inventário florestal sucessivo entre os anos de 2006 e 2010.

O procedimento de amostragem adotado foi - Sistemático Desencontrado (COCHRAN, 1977), lançando-se aproximadamente uma parcela retangular, contendo 60 covas, a cada 10 hectares de floresta. Em todas as parcelas, mediram-se os lados das mesmas com o emprego de trena, a circunferência a I,30 m do solo (CAP) de todos os fustes utilizando fita métrica, a altura total das dez primeiras árvores com fustes normais (sem bifurcação ou qualquer outro defeito) usando clinômetro digital de Häglöf e a altura das árvores dominantes, caso estas não estivessem entre as 10 alturas já mensuradas da parcela, conforme o conceito de Assmann, isto é, as alturas das 100 árvores mais grossas por hectare (ASSMANN, 1970). Além disso, as parcelas foram georreferenciadas para permitir o estudo variográfico. A estimativa do volume, uma das variáveis de interesse desse estudo, foi feita por meio da equação volumétrica, associada à equação hipsométrica, usada na estimativa das alturas das demais plantas dentro da parcela. A equação de volume foi obtida por idade, a partir da cubagem rigorosa referente a cada uma das medições realizadas na floresta, enquanto que a relação hipsométrica foi feita por parcela.

Com intuito de entender o comportamento geral dos dados, realizou-se uma análise exploratória dos mesmos. Nesta análise, foram determinadas as medidas de posição, dispersão, identificação de dados discrepantes e análise de tendência da disposição das amostras em relação à latitude e à longitude das variáveis: volume $(V)$, altura dominante média (HD) e incremento médio anual (IMA), em todas as idades de medição. As análises foram efetuadas utilizando o programa $R$ ( $R$ CORE TEAM, 20I3).

\section{Estudo variográfico e análise espacial para as diferentes idades}

Foram gerados, para as variáveis em estudo, os semivariogramas experimentais para cada idade de medição. A partir destes, foram ajustados pelo Método dos Mínimos Quadrados Ponderados, os modelos esférico, exponencial e gaussiano, descritos por Journel e Huijbregts (1978). Os ajustes foram feitos utilizando o programa R (R CORE TEAM, 20I3), por meio do pacote geoR (RIBEIRO JÚNIOR; DIGLLE, 200 I). A seleção do modelo se deu por meio do erro médio reduzido e desvio padrão do erro médio reduzido, estatísticas advindas da validação cruzada, descritos por McBratney e Webster (I986) e Cressie (1993).

A avaliação espacial foi efetuada por meio do semivariograma escalonado para cada característica dendrométrica em estudo e para cada idade de medição dos povoamentos de Eucalyptus sp trabalhada. 0 escalonamento gera o semivariograma com valores de semivariância padronizados entre 0 e I, permitindo comparar vários semivariogramas simultaneamente, tanto de variáveis distintas quanto da mesma variável ao longo do tempo. Mello et al. (2009) e Mello et al. (2005a) utilizaram de semivariogramas escalonados para comparar o comportamento espacial de características dendrométricas diferentes bem como para comparar a continuidade espacial de uma dada característica em diferentes tipos de parcelas, respectivamente. No caso do presente estudo, as variáveis foram analisadas para cada idade de plantio, de forma consecutiva, o que permitiu entender se o comportamento espacial destas é mantido ou alterado com a idade do povoamento, demonstrando mais uma possível aplicação da geoestatística com base no semivariograma escalonado.

Caso as curvas dos modelos ajustados para cada característica, dentro de cada idade, sejam semelhantes, a variável aleatória possuirá um comportamento semelhante no espaço e no tempo (VIEIRA et al., 1997). Assim, o semivariograma escalonado consiste de uma ferramenta importante quando se almeja avaliar o comportamento espacial de uma variável ao longo do tempo. Os semivariogramas escalonados foram gerados no programa R (R CORE TEAM, 2013), por meio do pacote geoR (RIBEIRO JÚNIOR; DIGLLE, 200I).

\section{RESULTADOS E DISCUSSÃO}

Pela análise exploratória dos dados de altura dominante $(\mathrm{m})$, volume $\left(\mathrm{m}^{3} / \mathrm{ha}\right)$ e incremento médio anual ( $\left.\mathrm{m}^{3} / \mathrm{ha} / \mathrm{ano}\right)$, obtiveram-se as estatísticas básicas, ou seja, média, desvio padrão e coeficiente de variação (CV) para cada idade de medição, apresentadas na Tabela I.

A altura dominante (HD) foi a característica dendrométrica que apresentou o menor coeficiente de variação em todas as idades avaliadas ao longo do tempo. Os dados de volume foram os que apresentaram a maior variabilidade entre as parcelas, com o $\mathrm{CV}$ variando de $14,20 \%$ a $21,44 \%$, demostrando uma considerável homogeneidade dessa característica dendrométrica no povoamento avaliado. É importante destacar que as variáveis dendrométricas tendem a apresentar menor 
variabilidade dos dados (CV) a partir da idade de 5,8 anos, demonstrando uma estabilização do crescimento das árvores. Outro aspecto importante é que o CV apresentou uma ligeira redução ao longo do tempo para as 3 variáveis avaliadas.

Tabela I Estatísticas descritivas, em cada idade de medição, para as características altura dominante (HD), volume $(\mathrm{V})$ e incremento médio anual (IMA).

Table I Descriptive statistics, at each age of measurement, for the characteristics dominant height (HD), volume $(\mathrm{V})$ and mean annual increment (IMA).

\begin{tabular}{|c|c|c|c|c|}
\hline \multirow{2}{*}{$\begin{array}{l}\text { Idade } \\
\text { (anos) }\end{array}$} & \multirow{2}{*}{ Estatística } & \multicolumn{3}{|c|}{ Característica dendrométrica } \\
\hline & & $\mathrm{HD}(\mathrm{m})$ & $\mathrm{V}\left(\mathrm{m}^{3} / \mathrm{ha}\right)$ & IMA $\left(\mathrm{m}^{3} / \mathrm{ha} / \mathrm{ano}\right)$ \\
\hline \multirow{3}{*}{2,7} & Média & 16,42 & 80,32 & 29,01 \\
\hline & $\begin{array}{l}\text { Desvio } \\
\text { padrão }\end{array}$ & $\mathrm{I}, 73$ & 17,22 & 5,66 \\
\hline & CV (\%) & 10,54 & 21,44 & $|9,5|$ \\
\hline \multirow{3}{*}{3,7} & Média & 21,00 & 150,4 & 40,38 \\
\hline & $\begin{array}{l}\text { Desvio } \\
\text { padrão }\end{array}$ & $\mathrm{I}, 6 \mathrm{I}$ & 24,45 & 6,20 \\
\hline & CV (\%) & 7,67 & 16,26 & 15,35 \\
\hline \multirow{3}{*}{4,8} & Média & 24,14 & 202,90 & 41,98 \\
\hline & $\begin{array}{l}\text { Desvio } \\
\text { padrão }\end{array}$ & 1,92 & 31,70 & 6,27 \\
\hline & CV (\%) & 7,95 & 15,62 & 14,94 \\
\hline \multirow{3}{*}{5,8} & Média & 26,86 & 253,6 & 43,39 \\
\hline & $\begin{array}{l}\text { Desvio } \\
\text { padrão }\end{array}$ & 2,02 & 36,02 & 6,01 \\
\hline & CV (\%) & 7,52 & 14,20 & 13,85 \\
\hline \multirow{3}{*}{6,8} & Média & 27,83 & 276,30 & 40,69 \\
\hline & $\begin{array}{l}\text { Desvio } \\
\text { padrão }\end{array}$ & 2,33 & 39,37 & 5,79 \\
\hline & CV (\%) & 8,37 & 14,25 & 14,23 \\
\hline
\end{tabular}

Em que: $H D=$ altura dominante $(m) ; V=$ volume $\left(m^{3} / h a\right) ; I M A=$ Incremento médio anual ( $\left.\mathrm{m}^{3} / \mathrm{ha} / \mathrm{ano}\right) ; \mathrm{CV}=$ coeficiente de variação $(\%)$

Não foram observadas tendências em relação à latitude e à longitude, nem presença de dados discrepantes em todas as idades avaliadas para os dados de altura dominante, volume e incremento médio anual. Segundo Ribeiro Júnior (1995), a presença de tendência nos dados pode provocar uma falsa estrutura de dependência espacial, ou mesmo fazer com que esta não seja detectada durante o estudo variográfico.

\section{Análise da continuidade espacial}

O modelo exponencial foi o modelo teórico que melhor se ajustou ao semivariograma experimental gerado para as características altura dominante, volume e incremento médio anual, em todas as idades avaliadas. O desempenho deste modelo foi confirmado pelo erro médio reduzido e desvio do erro médio obtidos pela validação cruzada.

Mello et al. (2009) e Mello et al. (2005a) verificaram, dentre os modelos avaliados, um desempenho superior do modelo exponencial para estudos envolvendo características dendrométricas de eucalipto. Oda-Sousa et al. (20I0) também selecionaram o modelo exponencial para caracterizar a continuidade espacial da área basal em uma floresta semidecidual. Os resultados encontrados por estes autores corroboram com a seleção do modelo exponencial para a caracterização da continuidade espacial das características dendrométricas avaliadas neste estudo, uma vez que os ajustes obtidos foram de boa qualidade e o modelo espacial tem sido empregado com êxito na análise de continuidade espacial do desenvolvimento de plantações de florestas tanto nativas quanto plantadas.

$\mathrm{Na}$ Tabela 2 encontram-se os parâmetros do modelo exponencial ajustado ao semivariograma experimental para as variáveis avaliadas em todas as idades de medição. Observa-se que todas as características avaliadas apresentam-se espacialmente estruturadas, ou seja, existe uma função estrutural com semivariância modelável.

O alcance do modelo espacial representa a distância máxima dentro da qual a característica apresenta-se espacialmente correlacionada (autocorrelação espacial). Observa-se que a característica que apresentou o maior alcance foi volume na primeira medição (2,7 anos). Para todas as características avaliadas, houve uma redução do valor do alcance com o aumento da idade, o que significa uma tendência de redução da distância dentro da qual existe autocorrelação espacial e, consequentemente, da própria existência de dependência espacial.

O efeito pepita $\left(\tau^{2}\right)$ refere-se a variação não explicada pelo componente espacial e normalmente está vinculada a um problema de amostragem em menores distâncias. No presente estudo, os maiores valores de efeito pepita foram verificados para a variável volume na idade de 2,7 anos ( $I^{\mathrm{a}}$ medição). Este comportamento diferenciado em relação às demais idades encontra subsídio no fato de que, no início da vida da floresta, há um forte efeito da micro variação do sítio e da competição entre as árvores por melhores condições edafoclimáticas (FOX;ADES; BI, 2000). Estes dois fatores, conjuntamente, afetam as variações aleatórias ocorridas 
Tabela 2 Parâmetros do semivariograma para o modelo exponencial nas 5 idades de medição, efeito pepita $\left(\tau^{2}\right)$, contribuição $\left(\sigma^{2}\right)$, alcance $(\phi)$ e o grau de dependência espacial (GDE\%, Cambardella, et all. 1994).

Table 2 Semivariogram parameters for the exponential model in 5 ages measurement, nugget effect $\left(\tau^{2}\right)$, contribution $\left(\sigma^{2}\right)$, range $(\phi)$ and spatial dependence (GDE\%, Cambardella, et all. 1994 ).

\begin{tabular}{ccccccc}
\hline Característica & & \multicolumn{5}{c}{ Idade (anos) } \\
\cline { 2 - 7 } dendrométrica & Parâmetro & 2,7 & 3,7 & 4,8 & 5,8 & 6,8 \\
\cline { 3 - 6 } & & 0,91 & 0,02 & 0,00 & 0,00 & 0,00 \\
$\mathrm{HD}(\mathrm{m})$ & $\tau^{2}$ & 3,19 & 2,81 & 3,96 & 4,16 & 5,09 \\
& $\sigma^{2}$ & 1096,87 & 370,87 & 272,07 & 324,41 & 279,00 \\
& $\phi$ & 77,8 & 99,3 & 100,0 & 100,0 & 100,0 \\
\hline \multirow{3}{*}{$\mathrm{G}\left(\mathrm{m}^{3} / \mathrm{ha}\right)$} & $\tau^{2}$ & 33,51 & 0,00 & 0,00 & 0,00 & 0,00 \\
& $\sigma^{2}$ & 342,97 & 691,20 & 1151,55 & 1608,85 & 1695,63 \\
& $\phi$ & 750,89 & 368,13 & 310,87 & 422,32 & 416,71 \\
& $\mathrm{GDE}(\%)$ & 91,1 & 100,0 & 100,0 & 100,0 & 100,00 \\
\hline \multirow{3}{*}{$\mathrm{MA}\left(\mathrm{m}^{3} / \mathrm{ha} /\right.$ ano $)$} & $\tau^{2}$ & 2,87 & 0,00 & 0,00 & 0,00 & 0,00 \\
& $\sigma^{2}$ & 35,76 & 45,20 & 45,10 & 41,01 & 33,54 \\
& $\phi$ & 459,98 & 343,06 & 267,02 & 330,87 & 298,67 \\
& $\mathrm{GDE}(\%)$ & 92,60 & 100,00 & 100,00 & 100,00 & 100,00 \\
\hline
\end{tabular}

Em que: $H D=$ altura dominante $(m) ; V=$ volume $\left(m^{3} \cdot h a^{-1}\right) ; I M A=$ Incremento médio anual $\left(\mathrm{m}^{3} / \mathrm{ha} / \mathrm{ano}\right)$.

nas características dendrométricas nesta idade. Estes fatores aumentam a aleatoriedade no desenvolvimento das árvores, aumentando as incertezas na micro-escala e, portanto, o efeito pepita. Nas demais características, o efeito pepita $\left(\tau^{2}\right)$ foi praticamente zero em todas as idades de medição, o que demonstra o elevado grau de dependência espacial (GDE), permitindo caracterizar as variáveis como altamente estruturadas. Vale ressaltar que o grau de dependência espacial foi inferior na primeira idade (2,7 anos) para as 3 características avaliadas. Esse fato está relacionado com o efeito pepita nesta idade ser maior que nas demais idades de medição.

Assis et al. (2009), avaliando a continuidade espacial de volume para eucalipto, verificou que nas idades de 2 a 3 anos houve forte dependência espacial, corroborando com os resultados obtidos nesse estudo para a variável volume por hectare, e evidenciando ainda que a estrutura de correlação espacial se manteve alta até a última medição. Por outro lado, Mello et al. (2009), trabalhando com dados de volume em eucalipto nas idades de 3 e 4 anos, não detectaram dependência espacial com a intensidade amostral de uma parcela a cada 10 hectares, o que demonstra que outros fatores além da espécie e da intensidade amostral influenciam no comportamento da estrutura de autocorrelação espacial. No presente estudo, com a mesma intensidade amostral o grau de dependência espacial para volume foi superior a $90 \%$, diferindo dos resultados obtidos por esses autores.
A presença de continuidade espacial em variáveis dendrométricas implica que as estimativas que envolvem essas características devem levar em consideração a estrutura de dependência espacial como garantia de uma maior precisão na estimativa das variáveis dendrométricas (MELLO et al., 2006). Observa-se ainda que o grau de dependência espacial é praticamente o mesmo ao longo dos anos para as 3 características avaliadas, evidenciando uma possível estrutura de dependência espacial similar ao longo do tempo destas características.

A fim de verificar o comportamento da estrutura espacial em função das idades (tempo) para cada característica em estudo, construiu-se os semivariogramas escalonados utilizando-se 0 modelo exponencial para as 5 idades de medição, como pode ser observado na Figura 1.

Verificou-se que o comportamento da estrutura de dependência espacial foi semelhante nas referidas idades para as 3 características dendrométricas. Apenas o modelo espacial para a medição aos 2,7 anos da variável volume ( $I^{\mathrm{a}}$ medição) e para a variável IMA, também na $I^{a}$ medição, sofreu um ligeiro afastamento em relação às demais medições.

Do ponto de vista do inventário florestal contínuo, atualmente adota-se uma parcela a cada 10 hectares em plantios de eucalipto. É interessante buscar uma alternativa para reduzir a intensidade amostral sem perda na precisão do acompanhamento do crescimento 
da floresta. Esta alternativa está associada à geração de estratos de produtividade o mais cedo possível, a fim de reduzir a intensidade amostral necessária para acompanhar o crescimento da floresta. Com base nos resultados do presente trabalho, seja a estratificação feita por meio da altura média das dominantes, volume ou incremento médio anual, esta deve acontecer na segunda medição (3,7 anos). A partir desta idade, é possível notar que a estrutura de continuidade espacial, dentro de cada uma das características para diferentes idades, foi muito semelhante. Este resultado implica na geração de estratos mais homogêneos à medida que a idade do povoamento aumenta, sendo um forte indicativo de estabilidade das parcelas dentro de cada estrato.

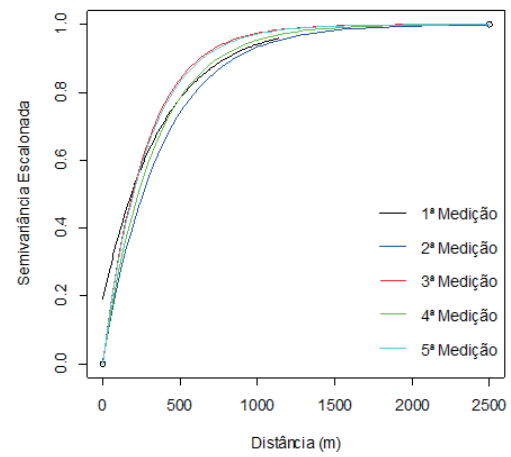

(a)

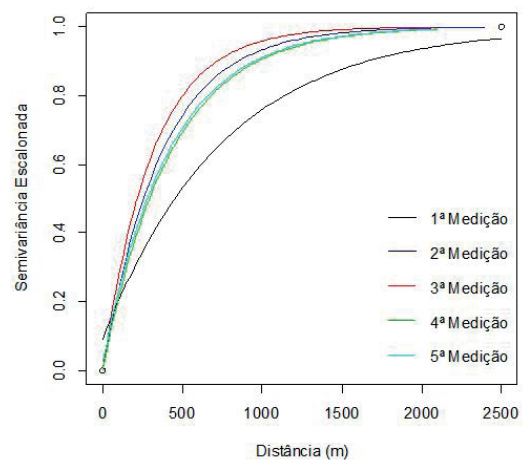

(b)
A estrutura espacial das características dendrométricas é semelhante em cada idade, exceto para a medição aos 2,7 anos, onde as curvas se diferenciam entre si quando comparadas com as demais medições. Assim, pode-se pensar em estratificação na segunda medição e utilizá-la ao longo da vida da floresta, permitindo redução de variabilidade e consequentemente custos no inventário florestal.

As características dendrométricas altura dominante média, volume e incremento médio anual apresentam-se estruturadas espacialmente em todas as idades de medição avaliadas, devendo esta continuidade espacial ser melhor explorada nas análises do inventário florestal a fim de se obter melhoria nas estimativas.

Figura I Semivariogramas escalonados do modelo exponencial para todas as idades de medição, para altura dominante (a), volume (b) e incremento médio anual (c).

Figure I Scaled semivariogram to the exponential model for all measurement ages dominant height (a), volume (b) and mean annual increment (c).

É importante ressaltar que não foram conduzidas análises de dependência temporal para as características dendrométricas dentro de cada estrato uma vez que os dados analisados correspondem a apenas 5 anos, impossibilitando uma análise de dependência temporal consistente. Contudo, entende-se que esta análise possa vir a ser realizada em consonância com a dependência espacial a fim de produzir informações no tocante à previsão do crescimento ao longo do tempo, embora o número de amostras temporais deve ser substancialmente maior.

\section{CONCLUSÕES}

modelo exponencial apresentou-se como o de melhor ajuste, com o menor efeito pepita para patamares muito próximos e a curva em si adequadamente se ajustando aos pontos do semivariograma.

O grau de dependência espacial se manteve ao longo dos anos para as características avaliadas, evidenciando que o crescimento temporal das árvores não afeta a dependência espacial destas características.

\section{REFERENCIAL BIBLIOGRÁFICO}

ALVARENGA, L. H. V.; MELLO, J. M. de; GUEDES, I. C. de L.; SCOLFORO, J. R. S. Desempenho da estratificação em um fragmento de cerrado stricto sensu utilizando interpolador geoestatístico. Cerne, Lavras, v. 18, p. $675-681,2012$.

ANTUNES, F. Z. Caracterização climática do Estado de Minas Gerais. Informe Agropecuário, Belo Horizonte, v.12, n. I38, p.9-13, jan. 1986.

ASSIS, A. L. de.; MELLO, J. M. de.; GUEDES, I. C. de L.; SCOLFORO, J. R. S.; OLIVEIRA, A. D. de. Development of a sampling strategy for young stands of Eucalyptus sp using geostatistics. Cerne, Lavras, v. 15, n. 2, p. 166I73, abr./jun. 2009.

ASSMANN, E. The principles of forest yield study. Oxford: Pergamon Press, 1970. $506 \mathrm{p}$.

CAMBARDELLA, C. A. et al. Field scale variability of soil properties in central lowa soils. Soil Science Society America Journal, Madison, v. 58, n. 9, p. I50I-I5II, Apr. 1994. 
COCHRAN, W. G. Sampling techniques. 3. ed. New York: J. Wiley, 1977. 555 p.

CRESSIE, A. G. Statistics for spatial data. New York: J. Wiley, 1993. $900 \mathrm{p}$.

FOX, J. C.; ADES, P. K.; BI, H. Stochastic structure and individualtree growth models. Forest Ecology and Management, v. I54, p. 26I-276, 200 I.

GUEDES, I. C. de L; MELLO, J. M. de; MELLO, C. R. de; OLIVEIRA, A. D. de; SILVA, S. T. da; SCOLFORO, J. R. S. Técnicas geoestatísticas e interpoladores espaciais na estratificação de povoamentos de Eucalyptus sp. Ciência Florestal, Santa Maria, v. 22, n. 3, p. 54I-550, jul.-set., 2012.

JOURNEL, A. G.; HUIJBREGTS, C. J. Mining geostatistics. London: Academic, 1978. $600 \mathrm{p}$.

KANEGAE JÚNIOR, H.; SCOLFORO, J. R. S.; MELLO, J. M. de; OLIVEIRA, A. D. de. Avaliação de interpoladores estatísticos e determinísticos como instrumento de estratificação de povoamentos clonais de Eucalyptus sp. Cerne, Lavras, v. I2, p. 123-136, 2006.

KANEGAE JÚNIOR, H.; MELLO, J. M. de.; SCOLFORO, J. R. S.; OLIVEIRA, A. D. de. Avaliação da continuidade espacial de características dendrométricas em diferentes idades de povoamentos clonais de Eucalyptus sp. Revista Árvore, Viçosa, v.3I, n.5, p.859-866, set./out. 2007.

McBRATNEY, A. B.; WEBSTER, R. Choosing functions for semivariograms of soil properties and fitting them to sampling estimates. European Journal of Soil Science, Oxford, v.37, n.4, p.617-639, Dec. 1986.

MELLO, J. M. de; BATISTA, J. L. F;; OLIVEIRA, M. S. de.; RIBEIRO JÚNIOR, P. J. Estudo da dependência espacial de características dendrométricas para Eucalyptus grandis. Cerne, Lavras, v. I I, n. 2, p. II3-126, abr./jun. 2005a.
MELLO, J. M. de.; BATISTA, J. L. F; RIBEIRO JÚNIOR, P. J.; OLIVEIRA, M. S. de. Ajuste e seleção de modelos espaciais de semivariograma visando à estimativa volumétrica de Eucalyptus grandis. Scientia Forestalis, Piracicaba, n.69, p.25-37, dez. 2005b.

MELLO, J. M. de.; OLIVEIRA, M. S. de.; BATISTA, J. L. F; RIBEIRO JÚNIOR, P. J.; KANEGAE JÚNIOR, H. Uso do estimador geoestatístico para predição volumétrica por talhão. Floresta, Curitiba, v. 36, n.2, p. 25I-260, maio/ ago. 2006.

MELLO, J. M. de ; DINIZ, F. S.; OLIVEIRA, A. D. de ; MELLO, C. R.; SCOLFORO, J. R. S.; ACERBI JÚNIOR, F. W. Continuidade Espacial para características dendrométricas (número de fustes e volume) em plantios de Eucalyptus grandis. Revista Árvore, v. 33, p. 185-194, 2009.

ODA-SOUZA, M.; BATISTA, J. L. F; RIBEIRO JUNIOR, P. J.; RODRIGUES, R. R. Comparação das estruturas de continuidade espacial em quarto formações florestais do estado de São Paulo. Revista Floresta, v.40, n.3, p.5 I5522, 2010.

R CORE TEAM. R: a language and environment for statistical computing. Vienna: R Foundation for Statistical Computing, 20 I3. Disponível em: < http://www.R-project.org>.

RIBEIRO JÚNIOR, P. J.; DIGGLE, P. J. geoR: a package for geostatistical analysis. R-NEWS, v. I, n.2, p.15-18, 2001.

RIBEIRO JÚNIOR, P. J. Métodos geoestatísticos no estudo da variabilidade espacial de parâmetros do solo. 1995. 99 p. Dissertação (Mestrado) - Escola Superior de Agricultura “Luiz de Queiroz”, Piracicaba.

VIEIRA, S.R.; NIELSEN, D.R.; BIGGAR, J.W.; TILLOTSON, P.M. The Scaling of semivariograms and the kriging estimation. Revista Brasileira de Ciência do Solo, v.21, p.525-533, 1997. 
